\title{
Métodos de Desinfección y Reutilización de Mascarillas con Filtro Respirador Durante la Pandemia de SARS-CoV-2
}

\author{
Decontamination Methods and Reuse of Filtering \\ Facepiece Respirators During SARS-CoV-2 Outbreak
}

Santos-López, Matías ${ }^{1,2}$; Jaque-Ulloa, Diego \& Serrano-Aliste, Sebastián ${ }^{1}$

SANTOS-LÓPEZ, M.; JAQUE-ULLOA, D. \& SERRANO-ALISTE, S. Métodos de desinfección y reutilización de mascarillas con filtro respirador durante la pandemia de SARS-CoV-2. Int. J. Odontostomat., 14(3):310-315, 2020.

RESUMEN: EI SARS-CoV-2 es un tipo de coronavirus con un alto grado de contagio entre pacientes y personal de salud, por lo que ha provocado la emergencia sanitaria más grande de los últimos años. Los equipos de protección personal (EPP) tienen como indicación un único uso, sin embargo, la escasez de estos ha llevado a buscar métodos para descontaminarlos y reutilizarlos, asegurando su disponibilidad. La evidencia presenta múltiples procedimientos para descontaminar mascarillas con filtro respirador tipo N95 (MFR N95), pero el método ideal debe inactivar al patógeno, asegurar la integridad del filtro respirador, el ajuste de la mascarilla y la salud del personal que la utilizará. La descontaminación con peróxido de hidrógeno vaporizado, irradiación germicida ultravioleta e incubación con calor húmedo se presentan como los métodos más eficaces contra distintos patógenos. Ante la creciente demanda de EPP y MFR N95, se sugiere realizar más estudios clínicos, que evalúen la eficacia de los métodos de descontaminación específicamente contra SARS-CoV-2 y se generen protocolos que permitan reutilizar con total seguridad este vital elemento para el personal de salud.

PALABRAS CLAVE: SARS-CoV-2, COVID-19, Equipo de Protección Personal, Mascarilla con Filtro Respirador.

\section{INTRODUCCIÓN}

Los coronavirus son una familia de virus que afectan al sistema respiratorio. En los últimos años han generado pandemias, como el Síndrome Respiratorio Agudo Severo en el 2003 (SARS-CoV) y el Síndrome Respiratorio de Medio Oriente (MERS-CoV) en el 2012 (Rothan \& Byrareddy, 2020).

En diciembre del 2019, las autoridades Chinas identificaron en Wuhan, un brote de neumonía atípica, con un agente causal hasta ese momento desconocido. Entre sus síntomas se encontraba la fiebre, signos radiológicos pulmonares y disnea. Se vinculó con el Mercado de mariscos de Wuhan, surgiendo la teoría de que fuera una enfermedad zoonótica (Lake, 2020).

El 8 de enero del 2020, el Centro Chino para el Control y la Prevención de Enfermedades (CCDC) anunció oficialmente el descubrimiento de un nuevo coronavirus, el COVID-19, considerándolo el responsable de los cuadros atípicos (Li et al., 2020). Con el aumento de los contagios, el 30 de enero, la Organización Mundial de la Salud declaró al Síndrome Respiratorio Agudo Severo 2 (SARS-CoV-2) como una emergencia mundial, y nombró oficialmente su infección como COVID-19 (Mahase, 2020; Meng et al., 2020).

A 4 meses del inicio de los primeros casos, el SARS-CoV-2 ha llegado a 185 países, contagiando a 2.382.064 personas y causando la muerte de 165.636 (Johns Hopkins University \& Medicine, 2020).

\section{¿QUÉ ES EL SARS-CoV-2?}

EI SARS-CoV-2 es un virus de RNA monocatenario positivo, perteneciente a la familia de los betacoronavirus. Los análisis filogenéticos muestran estrecho parentesco con los coronavirus presentes en murciélagos de herradura (97,8 \% de similitud) y en menor medida con el SARS-CoV (79 \%) y MERSCoV (50 \%) (Lu et al., 2020).

\footnotetext{
${ }^{1}$ Facultad de Odontología, Universidad Finis Terrae, Santiago, Chile.
}

${ }^{2}$ Asociación Nacional Científica de Estudiantes de Odontología, Chile. 
Se sugiere que tiene origen zoonótico, causado por el consumo de animales salvajes que lo hospedan (Rothan \& Byrareddy), para luego dar paso a un contagio entre humanos vía contacto directo o a través de secreciones respiratorias (Chan et al., 2020). No existe consenso con respecto a la media de incubación de la infección, pero se plantean tiempos entre 4 y 5,2 días, con un rango entre 0 y 24 días (Guan et al., 2020; Li et al.; Rothan \& Byrareddy).

La infección comenzaría con la unión del SARSCoV-2 a las células del hospedero a través del receptor de la enzima convertidora de angiotensina 2 (ECA2), lo que lleva a un cuadro clínico leve, con fiebre, tos y disnea, además de otros síntomas menos comunes como cefalea, hemoptisis, diarrea y linfopenia. En los cuadros clínicos más severos, se produce neumonía, leucocitosis y aumento del número de citoquinas pro-inflamatorias, lo que conlleva a la muerte del paciente (Rothan \& Byrareddy).

Actualmente se estudian distintos esquemas de tratamientos, entre los que destacan los antivirales remdesivir, lopinavir, ritonavir y oseltamivir, y combinaciones con fármacos como hidroxicloroquina y azitromicina (Lai et al., 2020; Amanat \& Krammer, 2020; Wang et al., 2020; Gautret et al., 2020). Por otra parte, los estudios enfocados en una vacuna, consideran que estará disponible entre 12 a 18 meses (Amanat \& Krammer).

\section{RIESGO DE CONTAGIO DEL PERSONAL DE SALUD}

La evidencia actual sugiere que el SARS-CoV-2 se transmite de persona a persona mediante contacto directo y fluidos respiratorios (Ong et al., 2020). Considerando que el manejo de los pacientes sospechosos y contagiados es realizado por el personal de salud, estos se encuentran con un riesgo de contagio extremadamente alto (Meng et al.).

La comprensión del mecanismo de transmisión del SARS-CoV-2, ha llevado a establecer protocolos de atención que obligan al uso de equipos de protección personal (EPP), los cuales permiten el manejo de pacientes sospechosos o contagiados, evitando la propagación viral a través del personal de salud (Honda \& Iwata, 2016).

\section{EQUIPO DE PROTECCIÓN PERSONAL}

Son los elementos artificiales utilizados por el personal de salud como barrera de protección contra ele- mentos y fluidos biológicos que pudiesen estar contaminados. Busca evitar la diseminación de contaminantes hacia las vías aéreas y otras zonas críticas del cuerpo. Se considera que las vías de ingreso más prevalentes corresponden a nariz y boca (Verbeek et al., 2019).

Existen múltiples protocolos para el manejo de infecciones respiratorias, pero en el caso del SARS-CoV2 , considerando su alta contagiosidad, se han extremado estas medidas para reducir al mínimo el riesgo de contagio (Feng et al., 2020).

Dentro de los EPP básicos se encuentran las mascarillas, escudos faciales, lentes, guantes y ropa impermeable (Hanoa \& Moen, 2016). Las mascarillas buscan ser una barrera física contra partículas o microorganismos que se encuentran en el aire. La MFR N95 es la más recomendada y utilizada para el manejo de infecciones respiratorias, ya que cubre de mejor manera las estructuras oronasales, creando un sello hermético con la piel, reduciendo al mínimo la posibilidad de contagio y evitando el paso de al menos un $95 \%$ de las partículas y microorganismos que se encuentran en el aire (The National Institute for Occupational Safety and Health, 2013; Chung et al., 2014; Smith et al., 2017; Centers for Disease Control and Prevention, 2020a).

\section{ESCASEZ DE EQUIPOS DE PROTECCIÓN PERSO- NAL (Organización Mundial de la Salud, 2020)}

La Organización Mundial de la Salud (OMS) ha advertido la creciente escasez de EPP, causado por el alto consumo por parte de los trabajadores de salud como también de la población en general, con el fin de evitar el contagio por SARS-CoV-2.

Según estimaciones de la OMS durante la pandemia del SARS-CoV-2, en un mes el personal de salud utilizará aproximadamente 89 millones de mascarillas, 76 millones de guantes y alrededor de 1,6 millones de gafas de seguridad.

A raíz de lo anterior, se hace necesario buscar alternativas que permitan mitigar la insuficiente cantidad de EPP. En especial de aquellas MFR N95, utilizadas por el personal médico durante el tratamiento de los pacientes contagiados con SARS-CoV-2.

\section{DESCONTAMINACIÓN Y REUTILIZACIÓN DE MFR N95}

Las MFR N95 son EPP desechables. Bajo condiciones normales no deben ser descontaminadas ni reutilizadas, sin embargo, la falta de EPP ha incentivado 
a investigar diversos métodos de desinfección, que permitan asegurar la disponibilidad de este crucial elemento para el personal de salud (Centers for Disease Control and Prevention, 2020b; Mackenzie, 2020).

Uno de los métodos para reutilizar este tipo de EPP se basa en la sobrevivencia natural del SARSCoV-2 en las superficies, que es alrededor de 72 horas en plásticos, metales y cartones (van Doremalen et al., 2020). Se sugiere alternar el uso de mascarillas tras haber sido almacenadas por más de 3 días, ya que permitiría inactivar el virus y que estos artículos puedan ser reutilizados. Cuando el stock de dichos EPP no es amplio, se deben evaluar otros métodos de descontaminación (Centers for Disease Control and Prevention, 2020b).

3M (Maplewood, MN, EE.UU), el mayor fabricante de MFR N95 en Estados Unidos, plantea que el proceso de descontaminación debe cumplir con cuatro objetivos (3M, 2020).

1. Ser efectivo contra el objetivo (SARS-CoV-2 en este caso).

2. No dañar el filtro respirador.

3. No afectar el ajuste de la mascarilla.

4. Ser seguro para la persona que utilizará la mascarilla.

La evidencia propone distintos métodos de descontaminación, como peróxido de hidrógeno vaporizado, irradiación germicida ultravioleta, vapor generado por microondas, incubación en calor húmedo, peróxido de hidrógeno líquido y óxido de etileno, los que se analizarán a continuación:

\section{PERÓXIDO DE HIDRÓGENO VAPORIZADO (PHV)}

Corresponde a ciclos de vaporizaciones de peróxido de hidrógeno, inyectados a baja presión y a distintas concentraciones en autoclaves específicos. El peróxido de hidrógeno forma radicales libres que permiten la eliminación de virus y bacterias de las superficies (Cornelia \& Warburton, 2017).

Se ha evaluado la descontaminación de MFR N95 con PHV, analizando la filtración y eliminación de patógenos. Se realizaron ciclos de PHV a baja presión, reduciendo e inactivando en un 99,99\% adenovirus, SARS-CoV, H1N1 y otros microorganismos, sin alterar la capacidad de filtrado de las mascarillas. (Viscusi et al., 2009; Goyal et al., 2014; Battelle Memorial Institute, 2016).
Actualmente se ha evaluado la efectividad del PHV en la eliminación del SARS-CoV-2, concluyendo que sería efectivo para la inactivación del virus y permitiendo la reutilización de la MFR N95 (Fischer et al., 2020).

El uso de PHV sería un método seguro y rápido en la descontaminación y reutilización de MFR N95, logrando mantener la integridad del filtro respiratorio y el ajuste de la mascarilla, eliminando al patógeno y permitiendo su reutilización por parte del personal de salud.

\section{IRRADIACIÓN GERMICIDA ULTRAVIOLETA (IGV)}

La IGV es un método que utiliza rayos UV de tipo $C$, que dañan el ADN y ARN impidiendo la replicación del patógeno (Hamzavi et al., 2020).

Se ha demostrado que la utilización de $1 \mathrm{~J} / \mathrm{cm}^{2}$ de UVC sobre MFR N95 contaminadas con influenza A (H1N1 - H5N1 - H7N9), MERS-CoV y SARS-CoV, permitiría la eliminación de muestras viables de dichos patógenos (Narla et al., 2020). Un reciente estudio realizado sobre MFR N95 infectadas con SARS-CoV2 demostró que la utilización de radiación UV de 260 $285 \mathrm{~nm}$ es efectiva como descontaminante, sin dañar el ajuste de la mascarilla ni el filtro respirador durante al menos 3 ciclos de descontaminación (Fischer et al.).

Considerando su capacidad de inactivar patógenos, no dañar el filtro respirador ni afectar el ajuste, la IGV se podría considerar un método seguro para reutilizar mascarillas.

\section{VAPOR GENERADO POR MICROONDAS (VGM)}

La técnica consiste en evaporar agua a través de microondas, generando vapor que tiene efecto biocida.

Heimbuch et al. (2011) analizaron la efectividad del VGM en mascarillas infectadas con $\mathrm{H} 1 \mathrm{~N} 1$, en un microondas con un reservorio de $50 \mathrm{ml}$ de agua a $1.250 \mathrm{~W}$ por 2 minutos. Si bien fue efectivo en la eliminación del virus, la mascarilla perdió ajuste en la zona de la nariz (Heimbuch et al.). Bergman et al. (2011) analizaron el ajuste de la mascarilla luego de tres ciclos de VGM, concluyendo que no existían modificaciones en el parámetro. Viscusi et al. concluyeron que la descontaminación con microondas debía ser la última opción por los daños que podía producir al filtro respirador. No se encontraron estudios que evaluaran la eficacia contra SARS-CoV-2. 
Por lo tanto, a pesar de que el VGM sería un método descontaminante efectivo, el posible daño al ajuste de mascarilla lo convierte en un método poco seguro.

\section{INCUBACIÓN EN CALOR HÚMEDO}

La incubación en calor húmedo genera partículas de agua que conducen la temperatura, desnaturalizando las proteínas de los patógenos y descontaminando.

Heimbuch et al. analizaron el calor húmedo como método de descontaminación contra $\mathrm{H} 1 \mathrm{~N} 1$, aplicándolo a $65^{\circ} \mathrm{C}$ con una humedad relativa (HR) de 85 $\%$ durante 30 minutos. Concluyeron que el 99,9 \% de los virus presentes en la superficie, incluyendo el $\mathrm{H} 1 \mathrm{~N} 1$, fueron completamente elimnados. Una de las razones por las cuales se sugiere que este método de desinfección es superior a los demás, se debe al ambiente producido por el calor húmedo, el cual se distribuye de forma homogénea a través de toda la MFR N95 mejorando su efectividad (Heimbuch et al).

De la misma forma, Bergman et al. aplicaron una incubación de calor húmedo en 3 modelos diferentes de MFR N95, a $60^{\circ} \mathrm{C}$, con HR de $80 \%$, eliminando los patógenos depositados en la superficie, y sin producir cambios relevantes en el ajuste de la mascarilla.

No se encontraron reportes de incubación en calor húmedo contra SARS-CoV-2, sin embargo, considerando su tasa de inactivación de patógenos, la ausencia de daño en el ajuste y el filtro respirador, y la seguridad para el personal de salud, sería un método a considerar para investigar su aplicación.

\section{PERÓXIDO DE HIDRÓGENO LÍQUIDO}

El peróxido de hidrógeno líquido genera la formación de radicales libres que llevan a la oxidación de los microorganismos que contactan con él.

Dembinski et al. documentaron que el peróxido de hidrógeno líquido durante un tiempo de exposición mayor a 30 minutos, a altas concentraciones, produce la inactivación y disminución virus como H1N1 y H2N3 (Dembinski et al., 2014). Bergman et al. evaluaron 6 modelos de MFR N95, realizando ciclos de inmersión en peróxido de hidrógeno líquido al $6 \%$ durante 30 minutos, con el fin de evaluar la capacidad de filtrado. Concluyeron que el líquido no afecta los filtros respiradores (Bergman et al.).
A pesar de su capacidad de inactivar patógenos y no alterar el filtro respirador, no se han realizado estudios que evalúen el ajuste posterior a la descontaminación.

\section{ÓXIDO DE ETILENO}

El óxido de etileno es un gas de uso frecuente en el proceso de esterilización de insumos termosensibles. Se ha comprobado su capacidad de descontaminación en MFR N95, siendo efectiva contra los patógenos, sin dañar el filtro respirador ni el ajuste de la mascarilla (Viscusi et al.).

Se ha limitado el uso de este gas descontaminante en EPP, ya que al contacto con piel y mucosa tendría tener un efecto carcinogénico o teratogénico. El proceso de ventilación en cámaras especiales es crítico, o de lo contrario, no se logra la total eliminación de las partículas gaseosas.

No existen estudios que comprueben la seguridad de las MFR N95 para la persona que la utilizará posterior al proceso de descontaminación con óxido de etileno (Centers for Disease Control and Prevention, 2020b; 3M).

\section{OTROS MÉTODOS}

Se han estudiado otros métodos de descontaminación además de los mencionados, tales como la aplicación de solución de etanol al $70 \%$ sobre la superficie de MFR N95, el cual inactivó rápidamente al SARS-CoV2 , sin embargo, produjo pérdida de la integridad de la MFR N95 y dañó al filtro respirador, razón por la cual no se recomienda utilizar (Fischer et al.).

También se ha reportado el uso de calor seco a $70^{\circ} \mathrm{C}$ por tiempos entre 10 a 60 minutos. Sin embargo, genera daño al filtro respirador luego de 2 ciclos de descontaminación, por lo cual se podría usar cuidadosamente, pero no como primera opción (Fischer et al.).

\section{CONCLUSIÓN}

La pandemia mundial por SARS-CoV-2 ha llevado a la escasez de EPP, y esto, a investigar métodos para descontaminar MFR N95 y asegurar su disponibilidad para el personal de salud. Existen múltiples métodos de descontaminación. El método ideal 
debe inactivar al patógeno, asegurar la integridad del filtro respirador, el ajuste de la mascarilla y la salud del personal que la utilizará. La descontaminación con peróxido de hidrógeno vaporizado, irradiación germicida ultravioleta e incubación con calor húmedo serían los métodos más eficaces. Se sugiere realizar más estudios que evalúen la eficacia de los métodos de descontaminación, y específicamente contra SARSCoV-2, para generar protocolos que permitan la reutilización de las MFR N95 con completa seguridad para el personal de la salud.

SANTOS-LÓPEZ, M.; JAQUE-ULLOA, D. \& SERRANOALISTE, S. Decontamination methods and reuse of filtering facepiece respirators during SARS-CoV-2 outbreak. Int. J. Odontostomat., 14(3):310-315, 2020.

ABSTRACT: SARS-CoV-2 is a type of coronavirus with a high risk of contagion among patients and health care workers $(\mathrm{HCW})$, being the cause of the largest health emergency in recent years. Personal Protective Equipment (PPE) are indicated for a single use. However, shortage has led to research new methods to decontaminate and reuse them, ensuring its availability. Evidence presents multiple procedures to decontaminate Filtering Facepiece Respirators type N95 (FFR), despite this, the ideal treatment should inactivate the pathogen, to ensure filter integrity, mask adjustments and the safety of who use this elements. Decontamination methods as vaporized hydrogen peroxide, ultraviolet germicidal irradiation and moist heat are the most effective procedures against different pathogens. Facing the rising demand of PPE and FFR, it's suggested to conduct researches to evaluate decontamination methods efficacy, and specifically against SARS-CoV-2, to generate protocols in ways to let to HCW reuse FFR N95 totally safe.

KEY WORDS: SARS-CoV-2, COVID-19, personal protective equipment, filtering facepiece respirators.

\section{REFERENCIAS BIBLIOGRÁFICAS}

3M. Decontamination Methods for 3M N95 Respirators. 3M, Technical Bulletin, Revision 4, April, 2020. Disponible en: https:/ /multimedia.3m.com/mws/media/18248690/decontaminationmethods-for-3m-n95-respirators-technical-bulletin.pdf

Amanat, F. \& Krammer, F. SARS-CoV-2 Vaccines: Status Report. Immunity, 52(4):583-9, 2020

Battelle Memorial Institute. Final Report for the Bioquell Hydrogen Peroxide Vapor (HPV) Decontamination for Reuse of N95 Respirators. Columbus, N95 FFR Decontamination for Reuse Final Report, 2016. Disponible en: https://www.fda.gov/media/ 136386/download

Bergman, M. S.; Viscusi, D. J.; Palmiero, A. J.; Powell, J. B. \& Shaffer, R. E. Impact of three cycles of decontamination treatments on filtering facepiece respirator fit. J. Int. Soc. Respir. Prot., 28(1):4859, 2011.
Centers for Disease Control and Prevention (CDC). Coronavirus Disease 2019. Using PPE. Atlanta, Centers for Disease Control and Prevention, 2020a. Disponible en: https://www.cdc.gov/ coronavirus/2019-ncov/hcp/using-ppe.html

Centers for Disease Control and Prevention (CDC). Coronavirus Disease 2019. Decontamination \& Reuse of Filtering Facepiece Respirators. Atlanta, Centers for Disease Control and Prevention, 2020b. Disponible en: https://www.cdc.gov/ coronavirus/2019-ncov/hcp/ppe-strategy/decontaminationreuse-respirators.html

Chan, J. F. W.; Yuan, S.; Kok, K. H.; To, K. K. W.; Chu, H.; Yang, J.; Poon, R. W. S.; Tsoi, H. W.; Lo, S. K. F.; Chan, K. H.; et al. A familial cluster of pneumonia associated with the 2019 nove coronavirus indicating person-to-person transmission: a study of a family cluster. Lancet, 395(10223):514-23, 2020.

Chung, S. J.; Ling, M. L.; Seto, W. H.; Ang, B. S. \& Tambyah, P. A. Debate on MERS-CoV respiratory precautions: surgical mask or N95 respirators? Singapore Med. J., 55(6):294-7, 2014.

Cornelia, R. \& Warburton, P. R. Assessing hydrogen peroxide vapor exposure from hospital sterilizers. J. Occup. Environ. Hyg., 14(9):150-7, 2017.

Feng, S.; Shen, C.; Xia, N.; Song, W.; Fan, M. \& Cowling, B. J. Rational use of face masks in the COVID-19 pandemic. Lancet Respir. Med., 2020. DOI: https://www.doi.org/10.1016/S22132600(20)30134-X

Fischer, R.; Morris, D. H.; van Doremalen, N.; Sarchette, S.; Matson, J.; Bushmaker, T.; Yinda, C. K.; Seifert, S.; Gamble, A.; Williamson, B.; et al. Assessment of N95 respirator decontamination and re-use for SARS-CoV-2. medRxiv, 2020. DOI: https://doi.org/10.1101/2020.04.11.20062018

Gautret, P.; Lagier, J. C.; Parola, P.; Hoang, V. T.; Meddeb, L.; Sevestre, J.; Mailhe, M.; Doudier, B.; Aubry, C.; Amrane, S.; et al. Clinical and microbiological effect of a combination of hydroxychloroquine and azithromycin in 80 COVID-19 patients with at least a six-day follow up: A pilot observational study. Travel Med. Infect. Dis., 101663, 2020. DOI: https://doi.org/10.1016/ j.tmaid.2020.101663

Goyal, S. M.; Chander, Y.; Yezli, S. \& Otter, J. A. Evaluating the virucidal efficacy of hydrogen peroxide vapour. J. Hosp. Infect., 86(4):255-9, 2014.

Guan, W. J.; Ni, Z. Y.; Hu, Y.; Liang, W. H.; Ou, C. Q.; He, J. X.; Liu, L.; Shan, H.; Lei, C. L.; Hui, D. S. C.; et al. Clinical characteristics of 2019 novel coronavirus infection in China. N. Engl. J. Med., 2020. DOI: https://doi.org/10.1056/NEJMoa2002032

Hamzavi, I. H.; Lyons, A. B.; Kohli, I.; Narla, S.; Parks-Miller, A.; Gelfand, J. M.; Lim, H. W. \& Ozog, D. Ultraviolet germicidal irradiation: possible method for respirator disinfection to facilitate reuse during COVID-19 pandemic. J. Am. Acad. Dermatol., 2020. DOI: https://doi.org/10.1016/j.jaad.2020.03.085

Hanoa, R. O. \& Moen, B. E. Ebola care and lack of consensus on personal protective respiratory equipment. Workplace Health Saf., 64(2):48-50, 2016.

Heimbuch, B. K.; Wallace, W. H.; Kinney, K.; Lumley, A. E.; Wu, C. Y.; Woo, M. H. \& Wander, J. D. A pandemic influenza preparedness study: use of energetic methods to decontaminate filtering facepiece respirators contaminated with H1N1 aerosols and droplets. Am. J. Infect. Control, 39(1):e1-9, 2011.

Honda, H. \& Iwata, K. Personal protective equipment and improving compliance among healthcare workers in high-risk settings. Curr. Opin. Infect. Dis., 29(4):400-6, 2016.

Johns Hopkins University \& Medicine. Coronavirus COVID-19 Global Cases by the Center for Systems Science and Engineering (CSSE) at Johns Hopkins University (JHU). Website, Johns Hopkins University \& Medicine, 2020. Disponible en: https:// coronavirus.jhu.edu/map.html 
Lai, C. C.; Shih, T. P.; Ko, W. C.; Tang, H. J. \& Hsueh, P. R. Severe acute respiratory syndrome coronavirus 2 (SARS-CoV-2) and coronavirus disease-2019 (COVID-19): The epidemic and the challenges. Int. J. Antimicrob. Agents, 55(3):105924, 2020.

Lake, M. A. What we know so far: COVID-19 current clinical knowledge and research. Clin. Med. (Lond.), 20(2):124-7, 2020.

Li, Q.; Guan, X.; Wu, P.; Wang, X.; Zhou, L.; Tong, Y.; Ren, R.; Leung, K. S. M.; Lau, E. H. Y.; Wong, J. Y.; et al. Early transmission dynamics in Wuhan, China, of novel coronavirusinfected pneumonia. N. Eng. J. Med., 382(13):1199-207, 2020.

Lu, R.; Zhao, X.; Li, J.; Niu, P.; Yang, B.; Wu, H.; Song, H.; Huang, B.; Zhu, N.; Bi, Y.; et al. Genomic characterisation and epidemiology of 2019 novel coronavirus: implications for virus origins and receptor binding. Lancet, 395(10224):565-74, 2020.

Mackenzie, D. Reuse of N95 masks. Engineering (Beijing), 2020. DOI: https://doi.org/10.1016/j.eng.2020.04.003

Mahase, E. China coronavirus: WHO declares international emergency as death toll exceeds 200. BMJ, 368:m408, 2020.

Meng, L.; Hua, F. \& Bian, Z. Coronavirus disease 2019 (COVID19): emerging and future challenges for dental and oral medicine. J. Dent. Res., 2020. DOI: https://doi.org/10.1177/ 0022034520914246

Narla, S.; Lyons, A. B.; Kohli, I.; Torres, A. E.; Parks-Miller, A.; Ozog, D. M.; Hamzavi, I. H. \& Lim, H. W. The Importance of the Minimum Dosage Necessary for UVC Decontamination of N95 Respirators during the COVID-19 Pandemic. Photodermatol. Photoimmunol. Photomed., 2020. DOI: https:/ /doi.org/10.1111/phpp.12562

Ong, S. W. X.; Tan, Y. K.; Chia, P. Y.; Lee, T. H.; Ng, O. T.; Wong, M. S. Y. \& Marimuthu, K. Air, Surface Environmental, and Personal Protective Equipment Contamination by Severe Acute Respiratory Syndrome Coronavirus 2 (SARS-CoV-2) From a Symptomatic Patient. JAMA, 2020. DOI: https://doi.org/ 10.1001/jama.2020.3227

Organización Mundial de la Salud. Shortage of Personal Protective Equipment Endangering Health Workers Worldwide. Ginebra, Organización Mundial de la Salud, 2020. Disponible en: https:/ /www.who.int/news-room/detail/03-03-2020-shortage-of-personal-protective-equipment-endangering-health-workersworldwide

Rothan, H. A. \& Byrareddy, S. N. The epidemiology and pathogenesis of coronavirus disease (COVID-19) outbreak. J. Autoimmun., 109:102433, 2020.

Smith, J.; MacDougall, C. C.; Johnstone, J.; Copes, R. A.; Schwartz, B. \& Garber, G. E. Effectiveness of N95 respirators versus surgical masks in protecting health care workers from acute respiratory infection: a systematic review and meta-analysis. CMAJ, 188(8):567-574, 2017.

The National Institute for Occupational Safety and Health (NIOSH). Respirator Awareness: Your Health May Depend On It. Personal Protective Equipment for Healthcare Workers. DHHS (NIOSH) Publication Number 2013-138 June 2013. Atlanta, Centers for Disease Control and Prevention, 2013. Disponible en: https://www.cdc.gov/niosh/docs/2013-138/

van Doremalen, N.; Bushmaker, T.; Morris, D. H.; Holbrook, M. G.; Gamble, A.; Williamson, B. N.; Tamin, A.; Harcourt, J. L.; Thornburg, N. J.; Gerber, S. I.; et al. Aerosol and surface stability of SARS-CoV-2 as compared with SARS-CoV-1. N. Engl. J. Med., 382(16):1564-7, 2020.

Verbeek, J. H.; ljaz, S.; Mischke, C.; Ruotsalainen, J. H.; Mäkelä, E.; Neuvonen, K.; Edmond, M. B.; Sauni, R.; Kilinc Balci, F. S. \& Mihalache, R. C. Personal protective equipment for preventing highly infectious diseases due to exposure to contaminated body fluids in healthcare staff. Cochrane Database Syst. Rev., 4:CD011621, 2019.
Viscusi, D. J.; Bergman, M. S.; Eimer, B. C. \& Shaffer, R. E. Evaluation of five decontamination methods for filtering facepiece respirators. Ann. Occup. Hyg., 53(8):815-27, 2009. Wang, M.; Cao, R.; Zhang, L.; Yang, X.; Liu, J.; Xu, M.; Shi, Z.; Hu, Z.; Zhong, W. \& Xiao, G. Remdesivir and chloroquine effectively inhibit the recently emerged novel coronavirus (2019-nCoV) in vitro. Cell Res., 30(3):269-71, 2020.

Dirección para correspondencia:

Matías Santos López

Facultad de Odontología

Universidad Finis Terrae

Santiago

CHILE

Email: m.santos@hotmail.cl

Recibido : 21-04-2020

Aceptado: 22-04-2020 